\title{
Evaluation of Candida Scoring Systems to Predict Early Candidemia: A Prospective and Observational Study at a Tertiary Care Hospital, Uttarakhand
}

\author{
Priyanka Gupta, Pratima Gupta, Biswaroop Chatterjee ${ }^{1}$, Garima Mittal ${ }^{1}$, Shashank Prateek ${ }^{2}$, Aroop Mohanty ${ }^{3}$ \\ Department of Microbiology, AllMS, Rishikesh, 'Department of Microbiology, Himalayan Institute of Medical Sciences, Jolly Grant, Dehradun, ${ }^{2}$ PDCC Infectious \\ Disease, Consultant Lab Medicine, Q Health Diagnostics, ${ }^{3}$ AllMS, Rishikesh, Uttarakhand, India
}

\section{Abstract}

Background: Candidemia in critically ill patients is usually a severe and life-threatening condition. Furthermore, due to its nonspecific presentation, it is difficult to diagnose leading to delayed treatment, prolonged hospitalization, and increased health-care costs with increase in morbidity and mortality. Objectives: In view of lack of data on "Candida scoring systems," this study was designed to evaluate the effectiveness of these scoring systems in predicting the development of candidemia among the Intensive Care Unit patients. Materials and Methods: The "Candida score" was calculated at the onset of systemic inflammatory response syndrome, sepsis, or shock. Various scoring systems were compared using the area under the receiver operating characteristic curve. Results: Among all three bedside risk scoring systems to predict candidemia both Leon score and Wenzel score offered significant discrimination between candidemic and noncandidemic patients with $P=0.000$ and 0.001 , respectively. The area under the curve for the scoring systems was $0.946(95 \%$ confidence interval [CI] $=0.89-1)$ and 0.818 (95\% CI $=0.687-0.949)$. Conclusion: Leon scoring system was found to have highest specificity, diagnostic accuracy, and positive likelihood ratio among all. Thus, we might conclude that a Leon score of $\geq 2.5$ was most suitable for diagnosis of candidemia with significant accuracy and shortening of turnaround time when compared to the gold standard of blood culture. To the best of our knowledge, this is the first report on the subject.

Keywords: Candida scoring systems, candidemia, Intensive Care Unit

\section{INTRODUCTION}

Bloodstream infections (BSIs) caused by various Candida spp. have been reported from all over the world and are a significant cause of morbidity and mortality in hospitalized patients, especially those in critical care units. Candida spp. are the most common cause of invasive fungal infections, accounting for $70 \%-90 \%$ of all invasive mycoses. ${ }^{[1]}$ Among the causes of nosocomial BSI, Candida spp. rank number four in the United States. ${ }^{[2]}$

The epidemiology of candidemia is complex and varies among the different patient care units. Its incidence varies between 0.5 and 1.4 per 10,000 patient-days in general wards and between 2 and 6.9 per 1,000 admissions in Intensive Care Units (ICUs). ${ }^{[3,4]}$ Various logistic regression analyses of data from observational studies suggest that Candida infection is an independent predictor of mortality among ICU patients..$^{[5,6]}$

\begin{tabular}{|l|l|}
\hline \multicolumn{3}{c|}{ Access this article online } \\
\hline Quick Response Code: & Website: \\
& www.ijccm.org \\
& \\
&
\end{tabular}

The incidence of candidemia in Asian countries is unclear due to the lack of multicentric studies. A study by Verma et al. from SGPGI, Lucknow, Uttar Pradesh, India, ranked Candida spp. eighth among all isolates from BSI. ${ }^{[7]}$ There are a lot of differences among the prevalence and incidence reports available from different parts of India.

Early diagnosis of invasive candidiasis remains a challenge, and criteria for starting empirical antifungal therapy in ICU patients remain poorly defined. Although risk factors for invasive candidiasis are well identified, these are so numerous and nondiscriminatory that most ICU patients cannot be

Address for correspondence: Dr. Priyanka Gupta, Department of Microbiology, AllMS, Rishikesh, Uttarakhand, India. E-mail: dr.priyankagupta60@yahoo.com

This is an open access article distributed under the terms of the Creative Commons Attribution-NonCommercial-ShareAlike 3.0 License, which allows others to remix, tweak, and build upon the work non-commercially, as long as the author is credited and the new creations are licensed under the identical terms.

For reprints contact: reprints@medknow.com

How to cite this article: Gupta P, Gupta P, Chatterjee B, Mittal G, Prateek S, Mohanty A. Evaluation of Candida scoring systems to predict early candidemia: A prospective and observational study at a tertiary care hospital, Uttarakhand. Indian J Crit Care Med 2017;21:830-5. 
effectively identified for the early institution of prophylactic antifungal treatment. ${ }^{[2]}$ Moreover, widespread administration of antifungal agents to ICU patients cannot be justified owing to the substantial increase in health-care costs, increased risk of the emergence of antimicrobial resistance in Candida spp., and occurrence of adverse drug reactions. ${ }^{[8]}$

In view of the importance of initiating early antifungal prophylaxis/therapy in high-risk patients, several authors have formulated clinical prediction rules or scoring systems to identify ICU patients at high risk of invasive candidiasis for whom initiation of empirical antifungal therapy could be justified. ${ }^{[9]}$ These scoring systems could help overcome this dilemma.

\section{Materials and Methods}

The study was carried out on a total of 75 patients, admitted to ICU of a tertiary care hospital over a period of 12 months. Participants fulfilling the inclusion criteria of the study (mentioned below) were recruited. Written informed consent was obtained from nearby relatives of all recruited patients. Clinical and epidemiological information obtained from these patients was recorded, bedside scoring was done, and Candida isolates recovered from blood samples were analyzed.

\section{Inclusion criteria}

Patient admitted in ICU with the following:

- Admission for $\geq 2$ days

- $\quad$ Systemic inflammatory response syndrome (SIRS): SIRS was defined as $\geq 2$ findings with temperature $>38^{\circ} \mathrm{C}$ or $<36^{\circ} \mathrm{C} /$ heart rate $>90 /$ respiratory rate $>20 /$ total leukocyte count $>12,000 / \mathrm{mm}^{3}$ or $<4000 / \mathrm{mm}^{3}$ or $>10 \%$ bands.

\section{Exclusion criteria}

- $\quad$ Age $<6$ years

- Pregnant and nursing women.
Blood culture was performed for documenting evidence of candidemia. Candidemia was defined as documentation of one or more blood culture that yielded a Candida spp. in a patient with consistent clinical manifestations. ${ }^{[10]}$ Unifocal colonization was defined as isolation of Candida from one focus, and multifocal colonization was defined as isolation of Candida from more than one noncontiguous foci, even with different Candida spp. Clinical sepsis was defined as features of SIRS along with source of infection. Bedside scoring was done for each patient. Each variable was given; 1 as present and 2 as clinical sepsis, and 0 as absent [Table 1]. Total risk score of each patient according to different scoring systems was calculated and further analyzed.

\section{Results}

The prevalence of candidemia among ICU patients with features of SIRS was $16 \%(n=12)$ in our study [Figure 1]. Out of 75 patients, blood culture was positive in $28(37.3 \%)$ patients

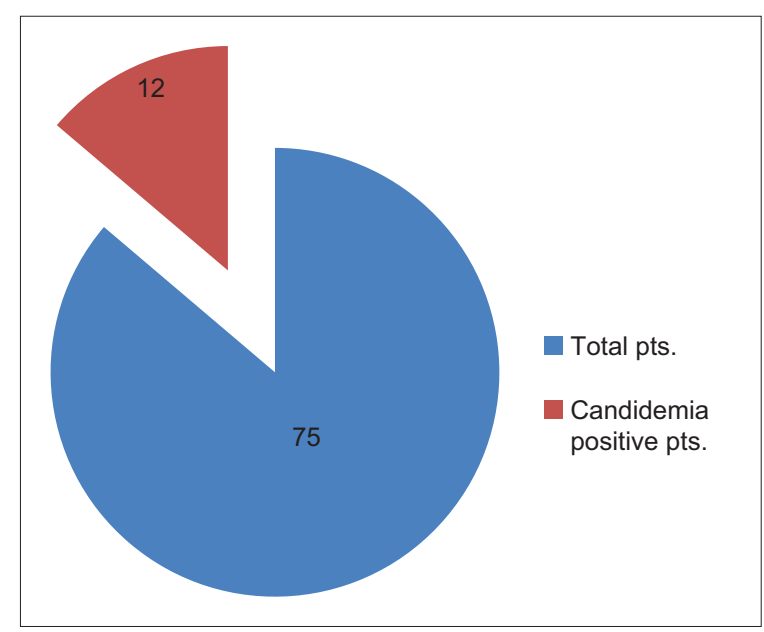

Figure 1: Prevalence of candidemia among total recruited patients

\begin{tabular}{|c|c|c|c|c|}
\hline $\begin{array}{l}\text { Candida scoring } \\
\text { systems }\end{array}$ & Candida risk factors & $\begin{array}{c}\text { Points } \\
\text { Present (1) }\end{array}$ & $\begin{array}{l}\text { Total score } \\
\text { Absent (0) }\end{array}$ & $\begin{array}{l}\text { Culture result (including } \\
\text { speciation and sensitivity } \\
\text { for culture-positive cases) }\end{array}$ \\
\hline Leon et al. & $\begin{array}{l}\text { Clinical sepsis } \\
\text { Surgery } \\
\text { Total parenteral nutrition } \\
\text { Multifocal colonization }\end{array}$ & & & \\
\hline Wenzel et al. & $\begin{array}{l}\text { Intravenous catheters } \\
\text { Hemodialysis } \\
\text { Antibiotic usage } \\
\text { Colonization with Candida }\end{array}$ & & & \\
\hline Shorr et al. & $\begin{array}{l}\text { Age }<65 \text { years } \\
\text { Temperature } \leq 98^{\circ} \mathrm{F} \\
\text { Severe altered mental state } \\
\text { Cachexia } \\
\text { Previous hospitalization within } 30 \text { days } \\
\text { Admitted from other health-care facility } \\
\text { Need for mechanical ventilation at the time of admission }\end{array}$ & & & \\
\hline
\end{tabular}


and was thus confirmed as cases of clinical sepsis. Out of these 28 isolates, 12 (42.9\%) were Candida species and $16(57.1 \%)$ were aerobic bacterial pathogens. Thus, 12 out of 75 patients were found to be positive for candidemia with prevalence rate of $16 \%$. Candida albicans was the most common species $(n=6,50 \%)$ isolated from total candidemic patients $(n=12)$, followed by Candida glabrata $(n=3,25 \%)$, Candida krusei $(n=2,16.6 \%)$, and Candida tropicalis $(n=1,8.3 \%)$. Majority of patients in the study group comprised of males $(n=57,76 \%)$. The male-to-female ratio was 3.2:1. Out of 57 male patients, $7(12.3 \%)$ had candidemia, whereas $5(27.7 \%)$ out of 18 female patients had candidemia [Figure 2]; however, this difference was statistically insignificant $(P=0.145)$. In the present study, the age of recruited patients ranged from 20 to 88 years with maximum number of patients were in the age group of $60-69$ years $(n=17,22.6 \%)$, followed by patients $(n=14,18.6 \%)$ in $40-49$ years' age group.

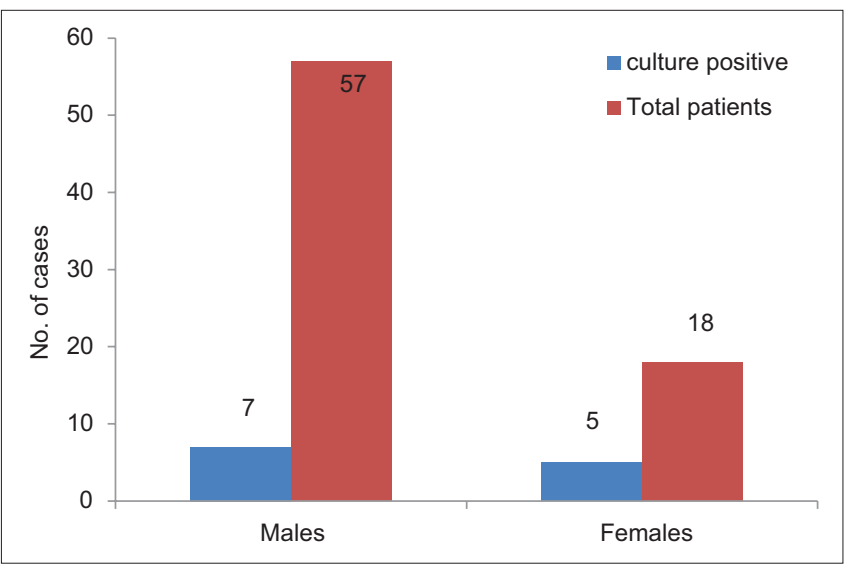

Figure 2: Comparison of gender-wise distribution among total and culture-positive patients
A total of 17 risk factors were identified among the recruited patients and analyzed for possible association with the development of candidemia. The maximum number of patients in our study group was on devices such as intravenous cannulae (82.6\%), endotracheal or tracheal tubes $(73.3 \%)$, and urinary catheters $(69.3 \%)$. Patients on mechanical ventilation $(41.3 \%)$ and those colonized by Candida at multifocal sites (34.6\%) comprised the next highest group. There were 26 patients $(34.6 \%)$ of clinical sepsis, 23 patients $(30.6 \%)$ with previous history of hospitalization, and 16 patients $(21.3 \%)$ each of prolonged ICU stay ( $>7$ days) and prolonged antibiotic usage ( $>7$ days) [Table 2]. Among these, six risk factors had a strong association with the occurrence of candidemia. Prolonged antibiotic usage was the most common risk factor associated with culture positivity for candidiasis $(P<0.00001$, $95 \%$ confidence interval $[\mathrm{CI}]=8.37-269.46)$, followed by prolonged ICU stay $(P=0.00024, \mathrm{CI}=3.35-56.3)$, multifocal colonization $(P=0.00025, \mathrm{CI}=2.90-74.2)$, clinical sepsis $(P=0.0024, \mathrm{CI}=1.96-33.58)$, recent surgery $(P=0.0194$, $\mathrm{CI}=1.53-35.46)$, and previous hospitalization within 30 days $(P=0.03, \mathrm{CI}=1.14-14.79)$ [Figure 3].

We constructed receiver operating characteristic curves [Figure 4] using SPSS 21.0 software (Armonk, NY: IBM Corp) for the three scoring systems analyzed in this study and observed that both Leon score and Wenzel score offered significant discrimination between candidemic and noncandidemic study participants, with $P=0.000$ and 0.001 , respectively. The area under the curve (AUC) for the scoring systems was $0.946(95 \% \mathrm{CI}=0.89-1)$ and $0.818(95 \% \mathrm{CI}=0.687-0.949)$ [Table 3]. However, Shorr score did not deliver such significant discrimination in our study $(P=0.398 ; \mathrm{AUC}=0.577 ; 95 \% \mathrm{CI}=0.405-0.750)$.

We also attempted to analyze the most suitable diagnostic cutoff for the three scoring systems and observed that a cutoff

\begin{tabular}{|c|c|c|c|c|c|}
\hline Risk factor & Total patients $(n=75)$ & Candida positive $(n=12)$ & $P$ & OR & $0.95 \% \mathrm{Cl}$ \\
\hline IV cannula & 62 & 10 & 0.65 & 1.05 & $0.20-5.5$ \\
\hline $\mathrm{ET} / \mathrm{TT}$ & 55 & 10 & 0.32 & 2 & $0.39-10.04$ \\
\hline Urinary catheter & 52 & 7 & 0.28 & 0.56 & $0.15-1.99$ \\
\hline Mechanical ventilation & 31 & 5 & 0.61 & 1.016 & $0.29-3.55$ \\
\hline Multifocal colonization (colonization index $\geq 0.5$ ) & 26 & 10 & 0.00025 & 14.68 & $2.90-74.2$ \\
\hline Clinical sepsis & 26 & 9 & 0.0024 & 8.11 & $1.96-33.58$ \\
\hline Previous hospitalization (within 30 days) & 23 & 7 & 0.03 & 4.11 & $1.14-14.79$ \\
\hline Cachexia & 18 & 3 & 0.59 & 1.06 & $0.255-4.45$ \\
\hline Prolonged ICU stay ( $\geq 7$ days) & 16 & 8 & 0.00024 & 13.35 & $3.35-56.3$ \\
\hline Prolonged antibiotic usage ( $\geq 7$ days) & 16 & 10 & $<0.00001$ & 47.5 & $8.37-269.46$ \\
\hline TPN & 15 & 3 & 0.44 & 1.41 & $0.33-6.04$ \\
\hline CVP & 14 & 3 & 0.39 & 1.57 & $0.36-6.7$ \\
\hline Admitted from other health care & 12 & 2 & 0.61 & 1.06 & $0.20-5.5$ \\
\hline Steroid usage & 10 & 3 & 0.19 & 2.66 & $0.58-12.2$ \\
\hline Hemodialysis & 10 & 3 & 0.19 & 2.66 & $0.58-12.2$ \\
\hline Severe altered mental status & 9 & 1 & 0.55 & 0.625 & $0.07-5.5$ \\
\hline Recent surgery & 8 & 4 & 0.0194 & 7.37 & $1.53-35.46$ \\
\hline
\end{tabular}

IV: Intravenous; ICU: Intensive Care Unit; TPN: Total parenteral nutrition; CVP: Central venous pressure; CI: Confidence interval; OR: Odds ratio; ET: Endotracheal tube; TT: Transtracheal tube 


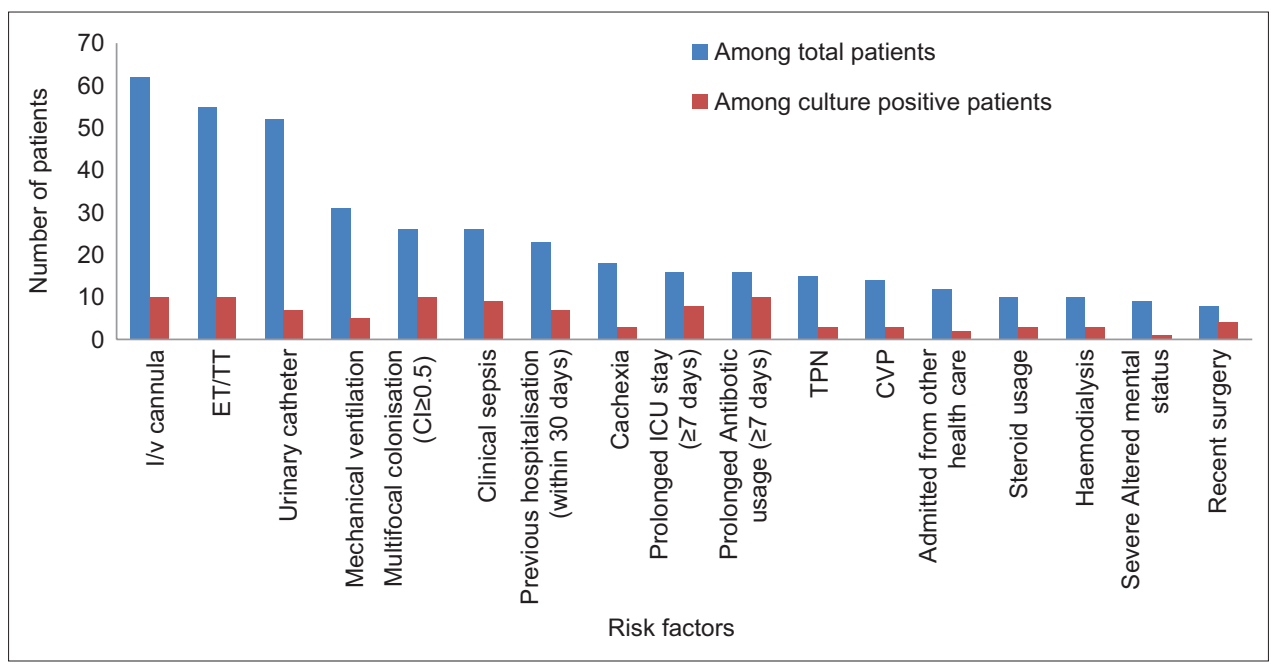

Figure 3: The most significant risk factors/predictive of candidemia

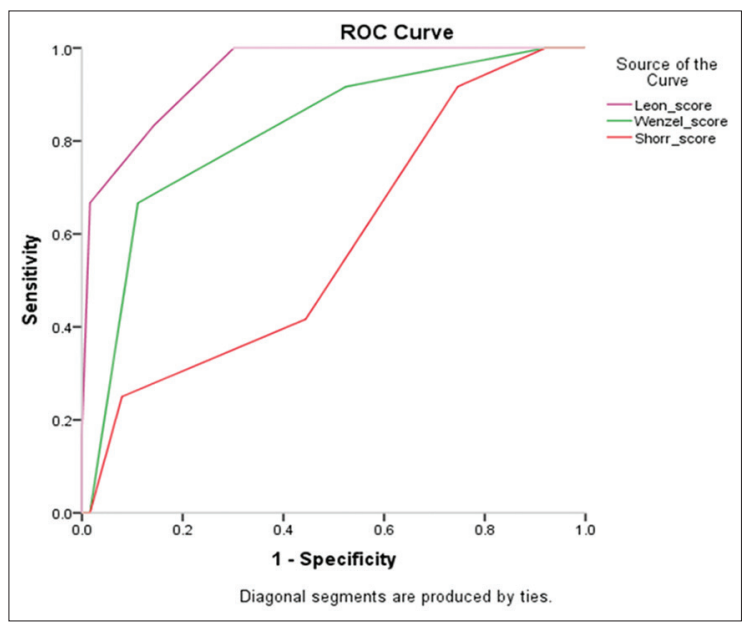

Figure 4: Receiver operating characteristic curve to compare between three risk scoring systems for candidemia

\begin{tabular}{lccccc}
\hline \multicolumn{3}{l}{ Table 3: Area under the curve for three scoring system } \\
\hline $\begin{array}{l}\text { Test result } \\
\text { variable(s) }\end{array}$ & Area & SE & $\boldsymbol{P}$ & \multicolumn{2}{c}{ Asymptotic 95\% Cl } \\
\cline { 4 - 6 } & & & & Lower bound & Upper bound \\
\hline Leon score & 0.946 & 0.029 & 0.000 & 0.890 & 1.000 \\
Wenzel score & 0.818 & 0.067 & 0.001 & 0.687 & 0.949 \\
Shorr score & 0.577 & 0.088 & 0.398 & 0.405 & 0.750 \\
\hline
\end{tabular}

CI: Confidence interval; SE: Standard error

of 2.5 and 1.5 for the Leon and Wenzel score, respectively, delivered the most favorable test characteristics using OpenEpi software (opensource.org/licenses) ${ }^{[11]}$ [Table 3].

\section{Discussion}

Candidemia is the fourth most common cause of hospital-acquired BSI. ${ }^{[12]}$ Prompt initiation of appropriate antifungal therapy is essential for managing invasive Candida infections; therefore, early diagnosis is a prerequisite for improving the prognosis of invasive candidiasis. However, timely laboratory confirmation of infection is often difficult; therefore, empirical antifungal therapy is often resorted to in ICU patients although the criteria for starting such therapy remain poorly defined. ${ }^{[13,14]}$ The prevalence of candidemia was determined and its relationship with the value of Candida score was analyzed. The clinical and epidemiological information was obtained and "Candida score" was calculated for each patient. This study brought about many interesting findings strengthening the fact that "Candida score" is an interesting tool to predict the early onset of candidemia in ICU patients. The prevalence of candidemia in our study was $16 \%$. In the present study, the age of recruited patients ranged from 20 to 88 years with maximum number of patients from 60 to 69 years $(22.6 \%)$, followed by age groups $40-49$ and 20-29 years. Candidemia is known to affect extremes of age due to building up of immune system in neonates and children and waning off immune response in elderly age group, but in our study, majority of patients were of middle-age group. The reason might be that these patients were admitted in ICU and were exposed to multiple risk factors, and neonates were not included in our study. The male outnumbered females in our study with male-to-female ratio being 3.1:1, which was similar to other studies by authors across the globe. Similar findings were reported by Leroy et al. and León et al. in their study. ${ }^{[10,13]}$

Among all three bedside risk scoring systems to predict candidemia, we found that both Leon score and Wenzel score offered significant discrimination between candidemic and noncandidemic patients. However, Shorr score did not deliver a similar significant discrimination in our study. We observed that a cutoff of 2.5 and 1.5 for the Leon and Wenzel score, respectively, gave the most suitable findings, and between these two scoring systems, Leon scoring system was found to have high specificity, diagnostic accuracy, and positive likelihood ratio [Table 4]. Although sensitivity and negative predictive value was lower than Wenzel, it was not statistically significant.

Like several other observers, the most significant independent risk factors associated with the occurrence of candidemia in our 
Table 4: Comparison of Leon and Wenzel scoring system

\begin{tabular}{|c|c|c|c|c|}
\hline \multirow[t]{2}{*}{ Parameter } & \multicolumn{2}{|c|}{ Leon score $\geq 2.5$} & \multicolumn{2}{|c|}{ Wenzel score $\geq 1.5$} \\
\hline & Estimate & Lower-upper (95\% Cls) & Estimate & Lower-upper (95\% CIs) \\
\hline Sensitivity & $83.33 \%$ & $55.2-95.3$ & $91.67 \%$ & $64.61-98.51$ \\
\hline Specificity & $85.71 \%$ & $75.03-92.3$ & $47.62 \%$ & $35.78-59.73$ \\
\hline Positive predictive value & $52.63 \%$ & $31.71-72.67$ & $25 \%$ & $14.57-39.44$ \\
\hline Negative predictive value & $96.43 \%$ & $87.88-99.02$ & $96.77 \%$ & $83.81-99.43$ \\
\hline Diagnostic accuracy & $85.33 \%$ & $75.62-91.61$ & $54.67 \%$ & $43.45-65.43$ \\
\hline Likelihood ratio of a positive test & 5.833 & $4.511-7.543$ & 1.75 & $1.623-1.887$ \\
\hline Likelihood ratio of a negative test & 0.1944 & $0.07254-0.5212$ & 0.175 & $0.02294-1.335$ \\
\hline Cohen's kappa (unweighted) & 0.5586 & $0.3414-0.7758$ & 0.1889 & $0.04273-0.3351$ \\
\hline
\end{tabular}

CIs: Confidence intervals

study were prolonged antibiotic usage $(P<0.00001)$, prolonged ICU stay $(P=0.00024)$, multifocal colonization $(P=0.00025)$, and recent surgery $(P=0.0194)$, clinical sepsis $(P=0.0024)$, and previous hospitalization within 30 days $(P=0.03) .{ }^{[15-18]}$

The use of broad-spectrum and prolonged antibiotic usage can lead to disruption of normal commensal flora of gut and cause colonization with Candida. Recent surgery, especially of gastrointestinal tract (GIT), was a common risk factor in our study and most of the other studies on candidemia since surgical procedures of the GIT might lead to mucosal disruption and cause seeding of the bloodstream by Candida spp. colonizing the gut. ${ }^{[19]}$ Gonzalez et al., Leon et al., and Blumberg et al. reported total parenteral nutrition (TPN) and renal replacement therapy as clinically significant risk factors; however, the same was not observed in our study. ${ }^{[20]}$ The reason can be due to relatively lower usage of TPN and less number of patients with chronic renal failure in ICU during our study period.

\section{Conclusion}

Thus, we might conclude that a Leon score of $\geq 2.5$ is most suitable for ruling in the diagnosis of candidemia with significant accuracy and shortening of turnaround time when compared to blood culture. To the best of our knowledge, comparison between scoring systems has not been done, yet thus, our findings maybe of significance for physicians to predict candidemia and initiate antifungal therapy to improve the clinical outcome of critically ill patients admitted in ICUs.

\section{Acknowledgments}

We would like to thank Dr. Debasis Biswas, Department of Microbiology, HOD and Professor, AIIMS, Bhopal, Madhya Pradesh, India.

\section{Financial support and sponsorship}

Nil.

\section{Conflicts of interest}

There are no conflicts of interest.

\section{References}

1. Lamagni TL, Evans BG, Shigematsu M, Johnson EM. Emerging trends in the epidemiology of invasive mycoses in England and Wales (1990-9).
Epidemiol Infect 2001;126:397-414.

2. Wisplinghoff $H$, Bischoff $T$, Tallent SM, Seifert H, Wenzel RP, Edmond MB, et al. Nosocomial bloodstream infections in US hospitals: Analysis of 24,179 cases from a prospective nationwide surveillance study. Clin Infect Dis 2004;39:309-17.

3. Kett DH, Azoulay E, Echeverria PM, Vincent JL; Extended Prevalence of Infection in ICU Study (EPIC II) Group of Investigators. Candida bloodstream infections in Intensive Care Units: Analysis of the extended prevalence of infection in Intensive Care Unit study. Crit Care Med 2011;39:665-70.

4. Marchetti O, Bille J, Fluckiger U, Eggimann P, Ruef C, Garbino J, et al. Epidemiology of candidemia in Swiss tertiary care hospitals: Secular trends, 1991-2000. Clin Infect Dis 2004;38:311-20.

5. Voss A, le Noble JL, Verduyn Lunel FM, Foudraine NA, Meis JF. Candidemia in Intensive Care Unit patients: Risk factors for mortality. Infection 1997;25:8-11.

6. Chen YC, Lin SF, Liu CJ, Jiang DD, Yang PC, Chang SC, et al. Risk factors for ICU mortality in critically ill patients. J Formos Med Assoc 2001;100:656-61.

7. Verma AK, Prasad KN, Singh M, Dixit AK, Ayyagari A. Candidemia in patients of a tertiary health care hospital from North India. Indian J Med Res 2003;117:122-8.

8. Nolla-Salas J, Sitges-Serra A, León-Gil C, Martínez-González J, León-Regidor MA, Ibáñez-Lucía P, et al. Candidemia in non-neutropenic critically ill patients: Analysis of prognostic factors and assessment of systemic antifungal therapy. Study Group of Fungal Infection in the ICU. Intensive Care Med 1997;23:23-30.

9. Paphitou NI, Ostrosky-Zeichner L, Rex JH. Rules for identifying patients at increased risk for Candidal infections in the surgical Intensive Care Unit: Approach to developing practical criteria for systematic use in antifungal prophylaxis trials. Med Mycol 2005;43:235-43.

10. León C, Ruiz-Santana S, Saavedra P, Almirante B, Nolla-Salas J, Alvarez-Lerma F, et al. A bedside scoring system ("Candida score") for early antifungal treatment in nonneutropenic critically ill patients with Candida colonization. Crit Care Med 2006;34:730-7.

11. Dean AG, Sullivan KM, Soe MM. OpenEpi: Open Source Epidemiologic Statistics for Public Health, Version. Available from: http://www.openEpi.com. [Last updated on 2013 Apr 06; Last accessed on 2014 Aug 20].

12. Shorr AF, Tabak YP, Johannes RS, Sun X, Spalding J, Kollef MH, et al. Candidemia on presentation to the hospital: Development and validation of a risk score. Crit Care 2009;13:R156.

13. Leroy G, Lambiotte F, Thévenin D, Lemaire C, Parmentier E, Devos $\mathrm{P}$, et al. Evaluation of "Candida score" in critically ill patients: A prospective, multicenter, observational, cohort study. Ann Intensive Care 2011;1:50.

14. Bassetti M, Mikulska M, Viscoli C. Bench-to-bedside review: Therapeutic management of invasive candidiasis in the Intensive Care Unit. Crit Care 2010;14:244.

15. Resende JC, de Resende MA, Saliba JL. Prevalence of Candida spp. In hospitalized patients and their risk factors. Mycoses 2002;45:306-12.

16. Chowta MN, Adhikari P, Rajeev A, Shenoy AK. Study of risk factors 
and prevalence of invasive candidiasis in a Tertiary care hospital. Indian J Crit Care Med 2007;11:67-73.

17. Xess I, Jain N, Hasan F, Mandal P, Banerjee U. Epidemiology of candidemia in a tertiary care centre of North India: 5-year study. Infection 2007;35:256-9.

18. Wenzel RP. Nosocomial candidemia: Risk factors and attributable mortality. Clin Infect Dis 1995;20:1531-4.

19. González de Molina FJ, León C, Ruiz-Santana S, Saavedra P; CAVA
I Study Group. Assessment of candidemia-attributable mortality in critically ill patients using propensity score matching analysis. Crit Care 2012; 16:R105.

20. Blumberg HM, Jarvis WR, Soucie JM, Edwards JE, Patterson JE, Pfaller MA, et al. Risk factors for Candidal bloodstream infections in surgical Intensive Care Unit patients: The NEMIS prospective multicenter study. The National Epidemiology of Mycosis Survey. Clin Infect Dis 2001;33:177-86. 